\title{
Making Sense of Developmental Dynamics
}

John P. Spencer

School of Psychology, University of East Anglia

\begin{abstract}
This commentary on the developmental dynamics special issue in Human Development identifies a set of common principles shared in the target articles. These common principles highlight how the dynamic approach has matured in the last 20 years into a mainstream developmental perspective. The commentary then discusses three core challenges facing the dynamic approach - the challenge of clear communication, the challenge of clearly defining learning in development, and the challenge of embracing the dynamics of neural systems. The commentary concludes with a charge inspired by Esther Thelen's work - to be relevant in the lives of children.
\end{abstract}

This special issue of Human Development focuses on the central question of our science - how development happens. The editors have assembled a set of leading scholars to outline how they think about development from a dynamic perspective, focusing on the processes that give rise to changes in behaviour through time. The authors have done an excellent job highlighting both their own perspectives as well as the philosophical and empirical implications of dynamic thinking. My charge is to reflect on the collection of papers, offering my own perspectives and highlighting some of the challenges ahead as we take developmental dynamics deeper into the $21^{\text {st }}$ century.

When I think back to the initial emergence of dynamical systems concepts in psychology in the 1980s and 1990s, I'm struck by how much of the dynamic approach was a 
reaction to other approaches. Dynamical systems approaches eschewed representation (van Gelder, 1998); the dynamic perspective was anti-boxology and anti-information processing (Port \& van Gelder, 1995); and the dynamic approach was decidedly anti-nativist (Thelen \& Smith, 1994). While many of these initial arguments live on in important ways today, it was always going to be the case that over time, the dynamic approach needed a frame-shift from what it is not, to what it is.

The past 20 years have provided that frame-shift, and this new perspective is reflected in the special issue. I would say there is important clarity in the special issue regarding which concepts are central to a developmental dynamics perspective. I highlight five examples to illustrate.

The importance of dynamics and complexity. All the authors embrace a dynamic view of development, but Van Geert's essay (this volume) struck home to me as he embraced dynamic systems theory as the backbone of any science of change. I couldn't agree more. Indeed, I think it is important for students in development to get exposure to formal training in dynamic systems theory, including some of the basic mathematics that underlies concepts such as an attractor. Van Geert has written eloquently on this topic, promoting the use of simple mathematical examples to help ground concepts that are fundamental to studying and understanding development (van Geert, 1994).

Van Geert also offers clarity on the type of system we are studying when we consider development. Here, he highlights the difference between a complicated system and a complex system. A complicated system has many parts, each of which can be understood by studying them in isolation. By contrast, in a complex system, those parts interact based on fundamental processes of coupling or coordination. This means that if you try to focus on one part in isolation, the whole tapestry of interactions falls apart. This 
forces a shift in how we do science toward studying the way the components interact rather than identifying which components are involved.

Stability and variability. Several authors highlighted the idea that variability and change are fundamental to how development works. Bickhard (this volume), in particular, emphasized that from a developmental dynamics perspective, change is the default; it is stability that requires explanation. This is a radical departure from prior views of development that emphasized the stable aspects of development. For instance, for decades, motor development was viewed through the lens of stability in the form of motor milestones. These were thought to be universal across children and cultures. More recently, however, researchers have documented the profound variability in motor development (Adolph \& Robinson, 2015), including variability in the timing of onset of each 'milestone' as well as variability in the ordering of the milestones. This has led to a shift in how we think about motor development, with a much stronger emphasis on how individuals assemble a sequence of skills through time-how they carve out stable action patterns amidst a sea of variability. Critically, as Adolph points out (this volume), this variability isn't noise; it is the stuff of creativity, helping children discover new stable action solutions.

Emergence and self-organization. The question of how new forms arise in development is central to our science - how the foetus grows fingers and toes; how infants develop new skills like reaching and crawling and walking; how children invent new ways of thinking, new concepts, new ways of taking another's perspective. How does something new arise in development if it wasn't there previously? The answer from a developmental dynamics perspective is emergence and self-organization. Although these concepts can sound almost magical, Van Geert highlights the immense literature in physics showing how emergence is a fundamental property of matter. These concepts are, of course, intricately 
bound to complexity, stability, and variability. Sometimes new forms appear out of nowhere because the system happens to stumble onto a new solution due to the inherent variability in complex systems. Put differently, because it is hard to do complex things in precisely the same way every time, the system can discover a new pattern on the fly as a new way of doing things spontaneously arises.

The question is whether 'accidental' learning like this is sufficient to explain aspects of development that are robust and seemingly 'universal' across children. Critical here is the observation that children don't all arrive at the same solution following the same path. That is, it is important not to confuse the regularity of the outcome with the regularity of the process each child takes to get there. To take a metaphor, if thousands crowd the beaches of Norfolk on a hot summer Saturday, we wouldn't conclude that beach-going was preprogrammed into the make-up of people in the east of England; rather, we would conclude that each person arrived at the beach through his or her own pathway, and we all happened to arrive at the same place and time because the conditions were ripe for a common outcome. The context and the interactions woven into how the system works set the stage for a robust solution to self-organize.

Autonomy. Children are autonomous systems; they seek out their own experiences in richly structured contexts; they explore according to both intrinsic and extrinsic goals. This property of development fundamentally changes the nature of change over time because each child's history is unique. Concretely, because children often explore the environment in different ways, they can learn different things. I've been struck by how profoundly this can affect learning. For instance, my colleagues and I have developed a model of word learning that learns word by autonomously exploring a visual world and forming associations between heard words and seen objects (Samuelson, Jenkins, \& 
Spencer, 2015). When we place this model in a word learning task, some models become 'strong' learners while other models become 'weak' learners. The kicker is that the models are all the same; the differences in performance at test arise from each model's history of autonomously looking and learning in the task. Conceptually, this means that what we consider 'individual differences' in the real task - strong vs weak learning - might actually emerge from processing in the task rather than be a difference that infants brought to the task prior to learning.

Other examples of autonomy were nicely highlighted in Di Paolo's work (this volume). For him, autonomy is central to sense-making - a child's striving to understand the external world. The challenges of 'sense making' are brought into sharp relief by work on developmental robotics. Here, researchers construct robotic models to understand the process by which new skills can be acquired through time. The nice thing about using a robot as a scientific tool is that you can look inside the robot to understand what was built in and what was emergent from the nature of how the robot autonomously explored the world and what it took away from this learning. Robots are also very useful because they force researchers to deal with the space-time details of sensori-motor exchanges between the robot and the world, including a world with social partners. One take-home message from this work is that knowledge or sense-making in an autonomous system is profoundly constrained by the step-by-step accumulation of sensori-motor experiences in a richly structured world within a richly structured body.

A focus on process. All of the articles in the special issue had a common focus on process - taking time seriously; embedding developmental research in dense data collected over multiple timescales. For instance, Adolph devoted an entire section to 'a process approach' with fantastic examples of how to implement a process perspective taken from 
her work on early motor development. Importantly, this focus on process was not just about changes within the child, but also emphasized the cascade of changes in the social contexts of development - as the child changes, so changes the world around the child. Ultimately, this focus on process is central to a dynamic view of development. As Van Geert highlights, a dynamic systems equation / model is an evolution rule that defines the process through time that governs how the system behaves.

Interim summary. Dynamic systems approaches to learning and development have a long history - the dynamic approach is no longer the new theory on the block. As such, it is exciting to see a sophisticated and coherent perspective emerging from this special issue, with common, shared concepts reflected in all of the essays. Importantly, these concepts were often grounded in concrete examples, highlighting that the dynamic approach is not just about theory, but also about how concepts are applied to empirical studies to shape how that work is done.

Challenges to the dynamic approach

The special issue highlights multiple shared concepts; it also brought to the foreground several challenges this perspective faces. In the sections below, I highlight three core challenges that I think will structure work in the coming decade.

Communication via language. Dynamical systems concepts are challenging, especially in a field that has been historically grounded in abstract concepts rather than mathematics. These challenges are compounded, as Adolph notes, by the language we use to describe development - concepts that, for instance, imply that we have fixed mental structures for perception and memory rather than dynamic processes of perceiving and remembering. Thus, as a field, we have a communication problem: how do we convey 
dynamic concepts through often non-dynamic language without recourse to mathematical formalisations?

There are at least two ways to push through this barrier. The first is by staying grounded through a judicious use of examples. If I survey the special issue, Adolph used many concrete examples, and her essay is crystal clear. Other essays used fewer concrete examples and I suspect readers will take away less from those essays. Thus, I challenge us all to 'up our game' and work hard to keep our writing concrete and grounded. Esther Thelen was a master at this. It's not a coincidence, then, that some of the most grounded discussion in the special issue was around her examples.

A second way to push through the communication barrier is to rely more on another type of language - mathematics. The dynamic approach has matured into a mainstream theory in our science. Thus, all students should receive graduate training in the dynamic approach including a basic introduction to the mathematics of dynamic systems theory. Several examples are readily available on this front to help students formalise their understanding of dynamical concepts (see resources at www.dynamicfieldtheory.org). What is needed now is up-take of these materials, as well as refinement of these materials to make them useful to a broad audience. I am a firm believer that a little bit of maths can go a long way.

Learning and development. A second challenge brought to the foreground in the special issue is the challenge of defining learning and development. Van Geert engages in a beautiful analysis of the semantics of the word 'development' going back to its roots in Dutch and German. This analysis leads him to define development in terms of dynamics, that is, as a force that gives rise to a capability, a possibility, a potential. Unfortunately, Van Geert spends less time on the concept of learning, so it is unclear whether he thinks there is 
an important distinction between these concepts. Di Paolo, similarly, blends these concepts freely throughout his essay.

Adolph does engage in this discussion, making a distinction between learning and development, learning versus development, or - her preference - learning in development. According to Adolph's analysis, development entails changes in the animal's body, brain, and nervous system, as well as changes in skills. By contrast, learning is what an animal must do to cope with or exploit changes in development that modify available affordances. This leads to behavioral flexibility - the ability to tailor ongoing behaviors to changes in local conditions. Interestingly, Bickhard falls on the opposite side of this debate, arguing that construction can take various forms and complexities, but that learning and development are not differing processes.

I like much of what Adolph has to say about learning in development. And we agree that something like growth of the body over development should be distinguished from learning. The tension comes when we think about the brain and nervous system-on this front, it is harder to separate learning and development because the brain shapes itself in complex ways over multiple timescales. For instance, structural brain development - often considered a form of physical growth - is influenced by experience-dependent processes (Forbes \& Gallo, 2017; Mount \& Monje, 2017) which are, of course, intricately linked to learning. This leads me to partially agree with Bickhard: changes in the brain can take various forms and complexities, making it hard to distinguish learning and development at the neural level. Ultimately, sorting through these details will require formal models of processes that create change over multiple timescales at the neural level, that is, dynamic systems models of neural learning and development. Critically, these will need to be embodied theories to connect up with changes in, for instance, the body over development. 
In addition to the debate about how to define learning and development, the authors also engage in a discussion about how to study learning and development. A key question here is what the unit of study should be - the individual? The individual in a laboratory context? The individual in a real-world context? The individual in a social context? In addition, should we be doing formal hypothesis testing or something more akin to discovery learning? On all of these fronts, there is little consensus across papers.

I think this divergence of perspectives is a good thing. For instance, my lab group engages in more hypothesis-testing work, often using constrained laboratory tasks inspired by information processing. At face value, these experiments are very different from the type of naturalistic exploration presented in, say, Adolph's essay. We take a different approach because we think laboratory tasks - though designed to isolate cognitive systems like working memory - give us leverage to understand how autonomous visual exploratory behaviors lead to autonomous learning. Critically, although we are interested in concepts like 'working memory', we always think about working memory in the context of an integrated system that includes populations of neurons (a brain) connected to a sensorimotor system (eyes and arms) embedded in a context. The fact that the context is wellstructured with precise timing and 'inputs' then becomes an advantage for us as we try to simulate how autonomous learning takes place in the lab (Perone, Simmering, \& Spencer, 2011; Perone \& Spencer, 2013b, 2014), as well as how this type of learning can extend out into the 'real' world (Perone \& Spencer, 2013a). The key point is that although my laboratory engages in a very different type of study from the studies presented by Adolph, we can each learn from the other because we share a common set of concepts. When it comes to studying development from a dynamic perspective, then, there doesn't have to be a right and a wrong way; diversity can lead to new insights. 
The dynamic brain. In the section above, I highlighted the ambiguity in what 'learning' and 'development' mean at the level of the brain. This is a launching point into the final challenge I was prompted to consider based on the target articles: there is a tension about how - and whether - to think about developmental dynamics in the context of neural dynamics. Bickhard takes the strongest stance, arguing that all dynamic processes must ultimately be realizable in neural terms; if a process cannot happen in the brain, then 'the model is false'. Di Paolo is also happy to embrace dynamics in neural terms. Adolph and Van Geert appear more agnostic.

A central challenge in thinking about developmental dynamics in neural terms is to have a language to use for such efforts. My colleagues and I have developed dynamic field theory (DFT) to push through this barrier (Schöner, Spencer, \& The DFT Research Group, 2015). DFT offers a rich set of concepts and a formal mathematical language - that can be visualized using computer simulations - to understand how populations of neurons give rise to perception, cognition, and action in a complex, embodied neural system. Working in psychology, my collaborators and I have focused on understanding the cognitive, learning, and developmental side of this challenge. Gregor Schoner's group has focused on how we can embed dynamic field models in robotic systems thereby interfacing with the real-time details of sensory and motor systems. The result is a rich theoretical and empirical landscape for thinking about embodied developmental dynamics.

Critically, we are taking the 'neural' aspects of this approach quite seriously. Initially, this included building concepts from empirical findings using primate neurophysiology. These efforts eventually led to tests of these concepts using multi-unit neurophysiology (Bastian, Riehle, Erlhagen, \& Schöner, 1998; Bastian, Schöner, Riehle, Schoner, \& Riehle, 2003; Erlhagen et al., 1999) and, more recently, voltage-sensitive dye imaging (Kozyrev, 
Eysel, \& Jancke, 2014). In all cases, empirical results from real brains successfully confirmed novel predictions about how neural systems work derived from dynamic field models. In recent work, we are extending this view of brain dynamics into non-invasive neuroscience techniques including fNIRS and fMRI (Buss \& Spencer, 2017; Wijeakumar, Ambrose, Spencer, \& Curtu, 2017). This is allowing us to directly test neural predictions of dynamic field models in young children and adults (Buss \& Spencer, 2017).

The next step in this line of work is to take development more seriously by testing longer-term and longitudinal predictions of dynamic field models at the neural level. Undoubtedly, our conceptualization of this landscape is limited; for instance, we have yet to implement any specific neural processes that concretely link to structural brain development including the myelination of the cortex. Nevertheless, we are collecting both structural and functional brain data within-subjects to generate the empirical database needed to understand how these processes relate to one another over multiple timescales. Ultimately, we hope to incorporate these different aspects of brain development into our theory to create an integrated theory of brain and behavioral development.

\section{Conclusions}

I end with a personal observation. As I read this collection of papers, I was struck by the many references to Esther Thelen's work and the clarity her examples provided. It is nearly 15 years since Esther passed away; I can't help imaging where our science would be if she had not lost her battle with cancer in 2004. And yet I think Esther would be pleased that developmental dynamics has become mainstream; that the ideas are still developing; that debate and discussion are thriving. 
That said, I think Esther would want to push us all to, above everything, be relevant. Dynamic systems theory can be opaque, abstract, and theoretical. Esther recognized this, and she worked hard to combat these tendencies in her writing. But she also recognized the importance of making dynamic systems theory relevant in the lives of children.

I think this is starting to happen. As one example, my colleagues and I have been using our insights into working memory, visual exploration, and brain development to try to understand how individual children are impacted by early adversity including poverty and undernutrition in India (Wijeakumar, Kumar, Reyes, Tiwari, \& Spencer, 2019). In this line of work, we are trying to build on a fundamental insight of a dynamic approach: if you understand the laws that govern change through time, successful intervention should be at your fingertips. This doesn't mean that everyone needs to do intervention work; there are many ways to be relevant. But the challenge remains to make this work and this theory impactful beyond academia. I look forward to seeing how we all embrace this challenge in the decades to come.

Acknowledgements

This work was supported by OPP1119415 from the Bill \& Melinda Gates Foundation awarded to John P. Spencer.

\section{References}

Adolph, K. E., \& Robinson, S. R. (2015). Motor Development. Handbook of Child Psychology and Developmental Science, 1-45. https://doi.org/10.1002/9781118963418.childpsy204

Bastian, A., Riehle, A., Erlhagen, W., \& Schöner, G. (1998). Prior information preshapes the 
population representation of movement direction in motor cortex. NeuroReport, 9(2), 315-319.

Bastian, A., Schöner, G., Riehle, A., Schoner, G., \& Riehle, A. (2003). Preshaping and continuous evolution of motor cortical representations during movement preparation. European Journal of Neuroscience, 18(7), 2047-2058. Retrieved from http://www.ncbi.nlm.nih.gov/pubmed/14622238

Buss, A. T., \& Spencer, J. P. (2017). Changes in frontal and posterior cortical activity underlie the early emergence of executive function. Developmental Science, (June 2017), 1-14. https://doi.org/10.1111/desc.12602

Erlhagen, W., Bastian, A., Jancke, D., Riehle, A., Schöner, G., Erlhange, W., ... Schoner, G. (1999). The distribution of neuronal population activation (DPA) as a tool to study interaction and integration in cortical representations. Journal of Neuroscience Methods, 94(1), 53-66. https://doi.org/10.1016/S0165-0270(99)00125-9

Forbes, T. A., \& Gallo, V. (2017). All Wrapped Up: Environmental Effects on Myelination. Trends in Neurosciences, 40(9), 572-587. https://doi.org/10.1016/j.tins.2017.06.009 Kozyrev, V., Eysel, U. T., \& Jancke, D. (2014). Voltage-sensitive dye imaging of transcranial magnetic stimulation-induced intracortical dynamics. Proceedings of the National Academy of Sciences, 111(37), 13553-13558.

https://doi.org/10.1073/pnas.1405508111

Mount, C. W., \& Monje, M. (2017). Wrapped to Adapt: Experience-Dependent Myelination. Neuron, 95(4), 743-756. https://doi.org/10.1016/j.neuron.2017.07.009

Perone, S., Simmering, V. R., \& Spencer, J. P. (2011). Stronger neural dynamics capture changes in infants' visual working memory capacity over development. Developmental Science, 14(6), 1379-1392. https://doi.org/10.1111/j.1467-7687.2011.01083.x 
Perone, S., \& Spencer, J. P. (2013a). Autonomous visual exploration creates developmental change in familiarity and novelty seeking behaviors. Frontiers in Psychology, 4(648), 121. https://doi.org/10.3389/fpsyg.2013.00648

Perone, S., \& Spencer, J. P. (2013b). Autonomy in Action: Linking the Act of Looking to Memory Formation in Infancy via Dynamic Neural Fields. Cognitive Science, 37(1), 1-60. https://doi.org/10.1111/cogs.12010

Perone, S., \& Spencer, J. P. (2014). The Co-Development of Looking Dynamics and Discrimination Performance. Developmental Psychology, 50(3), 837-852. https://doi.org/10.1037/a0034137

Port, R. F., \& van Gelder, T. (1995). Mind as Motion: Explorations in the Dynamics of Cognition (p. 590). p. 590. Cambridge, MA: MIT Press.

Samuelson, L. K., Jenkins, G. W., \& Spencer, J. P. (2015). Grounding cognitive-level processes in behavior: The view from dynamic systems theory. Topics in Cognitive Science, 7(2), 191-205. https://doi.org/10.1111/tops.12129

Schöner, G., Spencer, J. P., \& The DFT Research Group. (2015). Dynamic Thinking: A Primer on Dynamic Field Theory. New York, NY: Oxford University Press.

Thelen, E., \& Smith, L. B. (1994). A dynamic systems approach to the development of cognition and action. In Journal of Cognitive Neuroscience (Vol. 512). https://doi.org/10.1162/jocn.1995.7.4.512

van Geert, P. (1994). Dynamic systems of development. Change between complexity and chaos. New York: Harvester.

van Gelder, T. (1998). The dynamical hypothesis in cognitive science. Behavioral \& Brain Sciences, Vol 21, 615-665.

Wijeakumar, S., Ambrose, J. P., Spencer, J. P., \& Curtu, R. (2017). Model-based functional 
neuroimaging using dynamic neural fields: An integrative cognitive neuroscience approach. Journal of Mathematical Psychology, 76, 212-235.

https://doi.org/10.1016/j.jmp.2016.11.002

Wijeakumar, S., Kumar, A., Reyes, L. D., Tiwari, M., \& Spencer, J. P. (2019). Early adversity in rural India impacts the brain networks underlying visual working memory.

Developmental Science. https://doi.org/https://doi.org/10.1111/desc.12822 